\title{
T-Cube Web Tool for rapid detection of disease outbreaks in India and Sri Lanka
}

\author{
C. Weerasinghe \\ N. Waidyanatha \\ A. Adubrawski \\ M. Baysek \\ M. Ganesan \\ LIRNEAsia, Colombo, Sri Lanka. \\ Contact e-mail address: chamindusampath@gmail.com \\ eHealth Sri Lanka 2010,1(suppl.1):S40 \\ DOI: http://dx.doi.org/10.4038/sljbmi.v1i0.3693
}

Only the Abstract is available

\begin{abstract}
Motivated by existing gaps and inefficiencies in the paper-based manually processed disease surveillance and notification systems in India and Sri Lanka, the Real- Time Biosurveillance Programme (RTBP) introduces technology to health departments in Tamil Nadu, India and Sri Lanka, to test the question: "Can software programmmes that detect events in public health data, and mobile phones that collect health data and receive health alerts, enable effective identification and mitigation of disease outbreaks in near-real-time?" The processes involve digitising all clinical health records and analysing them in near real-time to detect emerging unusual patterns in data to forewarn health workers before the diseases reach epidemic states.

Health records from health facilities, namely the patient disease cases, syndromic, and demographic information, are transmitted through the mHealthSurvey mobile phone application 1 and fed in to the T-Cube data structure. T-Cube Web Interface (TCWI)2 is a browser-based software tool that uses the T-Cube data structure for fast retrieval and display of large volume multivariate time series and spatial information. Interface allows the user to execute complex queries quickly and to run various types of statistical tests on the loaded data. Detected emerging patterns of potentially epidemic events are then disseminated to health workers in the vulnerable and surrounding areas in the form of SMS, Email, and Web published alerts.

This paper considers utility and importance of TCWI in support of rapid detection and mitigation of bio- medical threats in developing countries. 1. Kannan T, Seebha R. Real-Time Biosurveillance Program: m-HealthSurvey Software Requirement Specifications [Internet]. 2009 Jan.Available from: http://lirneasia.net/wp-

content/uploads/2009/06/srs_mobile_application_rtbp_v20 .pdf 2. Ray S, Michalska A, Sabhnani M, Dubrawski A, Baysek M, Chen L, et al. T-Cube Web Interface: A Tool for Immediate Visualization, Interactive Manipulation and Analysis of Large Sets of Multivariate Time Series. AMIA Annual Symposium; 2008; Washington DC. 3. Gow G, Waidyanatha N. Common Alerting Protocol for Sahana Messaging Module: Real-Time Biosurveillance Program Software Requirement Specifications [Internet]. 2009. Available from: http://lirneasia.net/wpcontent/uploads/2009/05/Sahana-CAP-Msg-Mod-v0.2.pdf .
\end{abstract}

Keywords - T-Cube Web Tool, public health data, digitising, mHealthSurvey, RTBP 